\title{
Diffeomorphisms and holographic anomalies
}

\author{
C Imbimbo $\dagger$, A Schwimmer $\ddagger$ S Theisen $\S$ and S Yankielowicz\| \\ $\dagger$ Dipartimento di Fisica dell' Università di Lecce, Via Arnesano, I-73100, Lecce, Italy \\ \$ Department of Physics of the Complex Systems, Weizmann Institute, Rehovot 76100, Israel \\ § Sektion Physik, Universität München, Germany \\ \| School of Physics and Astronomy, Beverly and Raymond Sackler Faculty of Exact Sciences, \\ Tel-Aviv University, Ramat Aviv, Tel-Aviv 69978, Israel
}

Received 9 November 1999

\begin{abstract}
Using the relation between diffeomorphisms in the bulk and Weyl transformations on the boundary, we study the Weyl transformation properties of the bulk metric on-shell and of the boundary action. We obtain a universal formula for one of the classes of trace anomalies in any even dimension in terms of the parameters of the gravity action.
\end{abstract}

PACS number: 1125

\section{Introduction}

The AdS/CFT correspondence offers remarkable insights into non-perturbative phenomena in gauge theories [1]. Many of the proposed tests of the correspondence rely on the symmetry algebras being isomorphic.

Among the tests going beyond the mapping of the algebraic structure, the correct mapping of the trace anomalies is one of the most impressive [2-4]. On the supergravity side the correspondence involves a classical calculation: one solves the equation of motion using the metric at the boundary as an initial condition. The action evaluated for this classical solution gives the effective action in terms of the boundary metric. Taking a Weyl variation of the effective action gives the anomalous terms. An anomaly appears in a classical calculation due to the apparently infrared logarithmically divergent terms obtained when the action is evaluated with the classical solution. For the five-dimensional Einstein action with a cosmological constant the coefficients of the two independent trace anomaly structures match correctly the trace anomalies of the four-dimensional $N=4$ super-Yang-Mills theory calculated in the large- $N$ limit.

In this paper we study further the structure of this correspondence. Following Penrose [5] and Brown and Henneaux [6], we remark that the Weyl transformations of the boundary metric can be understood as a certain subgroup of the bulk diffeomorphisms. This observation allows us to derive a general transformation rule for the bulk metric when the boundary metric is changed by a Weyl transformation. In the following we will call it the 'PBH transformation'. The transformation does not use the solution of the equation of motion explicitly and, therefore, it is valid for a general bulk effective action including all the stringy corrections. We analyse the action evaluated for this general bulk metric and we uncover some universal properties of the trace anomaly which follow from it. In particular, the coefficient of the Euler structure present in any dimension (the so-called type A trace anomaly) has a universal dependence on 
the action, being given by the value of the action evaluated for the AdS solution. This allows us to calculate this particular trace anomaly for a general bulk action. The universal structure we find has general features reminiscent of the descent equations for the axial anomaly and probably has implications for the general understanding of the structure of trace anomalies, independent of the AdS/CFT correspondence.

In section 2 we discuss the algebraic structure of the PBH transformation. In section 3 we classify the terms in the effective action using the symmetry. The relation between the terms in the effective action and trace anomalies and a general formula for the type A trace anomaly are discussed in section 4 . The general conclusions we have reached and possible implications and open questions are discussed in the last section.

Most of the results of this paper have already been presented at Strings $99 \dagger$. Meanwhile several papers with some overlap have appeared [7].

\section{The PBH transformation}

Consider a manifold in $(d+1)$ dimensions with a boundary which is topologically $S_{d}$. Following Fefferman and Graham [8], one can choose a set of coordinates in which the $(d+1)$-dimensional metric has the form

$$
\mathrm{d} s^{2}=G_{\mu \nu} \mathrm{d} X^{\mu} \mathrm{d} X^{\nu}=\frac{l^{2}}{4}\left(\frac{\mathrm{d} \rho}{\rho}\right)^{2}+\frac{1}{\rho} g_{i j}(x, \rho) \mathrm{d} x^{i} \mathrm{~d} x^{j} .
$$

Here $\mu, v=1, \ldots, d+1$ and $i, j=1, \ldots, d$. The coordinates are chosen such that $\rho=0$ corresponds to the boundary. We will assume that $g_{i j}$ is regular at $\rho=0$, where $g_{i j}(x, \rho=0)$ is the boundary metric.

We now look for those $(d+1)$-dimensional diffeomorphisms which leave the form of the metric invariant. We make the ansatz

$$
\begin{aligned}
& \rho=\rho^{\prime} \mathrm{e}^{-2 \sigma\left(x^{\prime}\right)} \simeq \rho^{\prime}\left(1-2 \sigma\left(x^{\prime}\right)\right), \\
& x^{i}=x^{\prime i}+a^{i}\left(x^{\prime}, \rho^{\prime}\right) .
\end{aligned}
$$

The $a^{i}\left(x^{\prime}, \rho^{\prime}\right)$ are infinitesimal, and are restricted by the requirement of form invariance of the metric. We will work to $\mathcal{O}\left(\sigma, a^{i}\right)$. We insert (2.2) into (2.1) and require that the $\mathrm{d} x^{\prime} \mathrm{d} \rho^{\prime}$ components of the metric vanish. This gives

$$
\partial_{\rho} a^{i}=\frac{1}{2} l^{2} g^{i j} \partial_{j} \sigma .
$$

With the boundary condition $a^{i}(x, \rho=0)=0$ this integrates to

$$
a^{i}(x, \rho)=\frac{1}{2} l^{2} \int_{0}^{\rho} \mathrm{d} \rho^{\prime} g^{i j}\left(x, \rho^{\prime}\right) \partial_{j} \sigma(x) .
$$

Performing the diffeomorphism defined by (2.2), $g_{i j}$ will generally transform:

$$
\delta g_{i j}(x, \rho)=2 \sigma\left(1-\rho \partial_{\rho}\right) g_{i j}(x, \rho)+\nabla_{i} a_{j}(x, \rho)+\nabla_{j} a_{i}(x, \rho) .
$$

The covariant derivatives are with respect to the metric $g_{i j}(x, \rho)$ where $\rho$ is considered as a parameter.

Equations (2.2), (2.4) and (2.5) define the PBH transformation, i.e. a subgroup of bulk diffeomorphisms which leave the metric in the form (2.1) and which on the boundary reduce to a Weyl transformation.

$\dagger$ http://strings99.aei-potsdam.mpg.de/cgi-bin/viewit.cgi?speaker=Theisen 
We assume that $a^{i}(x, \rho)$ and $g_{i j}(x, \rho)$ have power-series expansions in the vicinity of $\rho=0$, i.e. we write

$$
a^{i}(x, \rho)=\sum_{n=1}^{\infty} a_{(n)}^{i}(x) \rho^{n}
$$

and

$$
g_{i j}(x, \rho)=\sum_{n=0}^{\infty} g_{(n) i j}(x) \rho^{n} .
$$

For a general bulk metric the coefficients in the expansion are arbitrary. If, however, the metric is the solution of an equation of motion, the coefficients of the expansion are all expressible in terms of the boundary metric $g_{(0) i j}$. This result was proven by Fefferman and Graham for the equation of motion following from the Einstein action with a cosmological term; we will assume that it holds for any action which admits an AdS solution. For $d$ an even integer, the expansion for $g$ also has logarithmic terms. Using dimensional regularization (i.e. $d$ noninteger) these terms are absent in the Fefferman-Graham case; we assume that this feature also holds for the general case. The coefficients in the expansion of $g_{i j}(x, \rho)$ are covariant tensors built from the boundary metric $g_{(0) i j}(x)=g_{i j}(x, \rho=0)$, since general covariance in $d$ dimensions is explicitly kept. Moreover, by a simple scaling argument $g_{(n)}$ contains $2 n$ derivatives with respect to the $x$ variables. The PBH transformation implies that the behaviour of $g_{(n)}$ under a Weyl transformation of the boundary metric is known. This determines to a great extent the expressions from $g_{(n)}$ in terms of covariant tensors built of $g_{(0) i j}$. We now perform the calculation outlined above.

We first express the $a_{(n)}$ in terms of the $g_{(n)}$. The first few terms are

$$
\begin{aligned}
& a_{(1)}^{i}=\frac{1}{2} l^{2} g_{(0)}^{i j} \partial_{j} \sigma, \\
& a_{(2)}^{i}=-\frac{1}{4} l^{2} g_{(1)}^{i j} \partial_{j} \sigma, \\
& a_{(3)}^{i}=\frac{1}{6} l^{2}\left[g_{(1)}^{i k} g_{(1) k}^{j}-g_{(2)}^{i j}\right] \partial_{j} \sigma, \\
& a_{(4)}^{i}=\frac{1}{8} l^{2}\left[-g_{(3)}^{i j}+g_{(1)}^{i k} g_{(2) k}^{j}+g_{(2)}^{i k} g_{(1) k}^{j}-g_{(1)}^{i}{ }_{k} g_{(1)}{ }_{k}{ }_{l} g_{(1)}^{l j}\right] \nabla_{j} \sigma .
\end{aligned}
$$

The variations of $g_{(n)}$ under a Weyl transformation of $g_{(0) i j}$ are easily obtained by combining (2.2), (2.4) and (2.5) with the expansion (2.7). Again we give just the first few terms:

$$
\begin{aligned}
& \delta g_{(0) i j}=2 \sigma g_{(0) i j}, \\
& \delta g_{(1) i j}=\stackrel{(0)}{\nabla}_{i} a_{(1) j}+\stackrel{(0)}{\nabla}_{j} a_{(1) i}, \\
& \delta g_{(2) i j}=-2 \sigma g_{(2) i j}+a_{(1)}^{k} \stackrel{(0)}{\nabla}_{k} g_{(1) i j}+\stackrel{(0)}{\nabla}_{i} a_{(2) j}+\stackrel{(0)}{\nabla}_{j} a_{(2) i}+g_{(1) i k} \stackrel{(0)}{\nabla}_{j} a_{(1)}^{k}+g_{(1) j k} \stackrel{(0)}{\nabla}_{i} a_{(1)}^{k} .
\end{aligned}
$$

The covariant derivative $\stackrel{(0)}{\nabla}_{i}$ is with respect to $g_{(0)}$. These equations can be integrated with respect to $\sigma$. For the first two non-trivial terms in the expansion (2.7) we find

$$
\begin{aligned}
& g_{(1) i j}=\frac{l^{2}}{d-2} {\left[R_{i j}-\frac{1}{2(d-1)} R g_{(0) i j}\right], } \\
& g_{(2) i j}=c_{1} l^{4} C_{i j k l} C^{i j k l} g_{(0) i j}+c_{2} l^{4} C_{i k l m} C_{j}{ }^{k l m}+\frac{l^{4}}{d-4}\left\{-\frac{1}{8(d-1)} \nabla_{i} \nabla_{j} R\right. \\
& \quad+\frac{1}{4(d-2)} \nabla^{2} R_{i j}-\frac{1}{8(d-1)(d-2)} \nabla^{2} R g_{(0) i j}-\frac{1}{2(d-2)} R^{k l} R_{i k j l}
\end{aligned}
$$




$$
\begin{aligned}
& +\frac{d-4}{2(d-2)^{2}} R_{i}{ }^{k} R_{j k}+\frac{1}{(d-1)(d-2)^{2}} R R_{i j}+\frac{1}{4(d-2)^{2}} R^{k l} R_{k l} g_{(0) i j} \\
& \left.-\frac{3 d}{16(d-1)^{2}(d-2)^{2}} R^{2} g_{(0) i j}\right\} .
\end{aligned}
$$

Here the curvature $R$, the Weyl tensor in $d$ dimensions $C$ and the covariant derivative $\nabla$ are all those of the boundary metric $g_{(0)} \dagger$.

Starting from $g_{(2)}$, there are solutions to the homogeneous equations, i.e. curvature invariants which transform homogeneously under Weyl transformations. For example, there are two free parameters for $g_{(2)}$. Of course we have complete agreement with the explicit calculation of [3].

We stress that the above expressions do not assume any specific form of the action. The dependence on the action enters only through the arbitrary coefficients of the homogeneous terms. Our goal will be to extract the universal (i.e. independent on the arbitrary coefficients) information about the trace anomalies.

\section{The PBH transformation and the effective boundary action}

We now study the implications of the symmetry defined above for a general gravitational action $S$ which is invariant under $(d+1)$-dimensional diffeomorphisms:

$$
S=\frac{1}{2 \kappa_{d+1}^{2}} \int \mathrm{d}^{d+1} X \sqrt{G} f(R(G)),
$$

where $f$ is a local function of the curvature and its covariant derivatives. For the application we have in mind, we must choose $f(R)$ such that $A d S_{d+1}$ with radius $l$ is a solution of the equations of motion. If we insert (2.1) into (3.1) we obtain an expression of the form

$$
2 \kappa_{d+1}^{2} S=\frac{1}{2} l \int \mathrm{d} \rho \mathrm{d}^{d} x \rho^{-(d / 2)-1} \sqrt{g_{(0)}(x)} b(x, \rho),
$$

where the specific form of $b(x, \rho)$ does depend on $f(R)$.

Since the integrand is a scalar under diffeomorphisms, the action satisfies

$$
\begin{aligned}
2 \kappa_{d+1}^{2} S & =\frac{1}{2} l \int \mathrm{d} \rho \mathrm{d}^{d} x \rho^{-(d / 2)-1} \sqrt{g_{(0)}(x)} b(x, \rho) \\
& =\frac{1}{2} l \int \mathrm{d} \rho^{\prime} \mathrm{d}^{d} x^{\prime} \rho^{\prime-(d / 2)-1} \sqrt{g_{(0)}^{\prime}\left(x^{\prime}\right)} b^{\prime}\left(x^{\prime}, \rho^{\prime}\right) .
\end{aligned}
$$

On a solution of the equation of motion $b(x, \rho)$ becomes a functional of $g_{(0)}$ and therefore using (3.3) for the diffeomorphisms (2.2) we obtain its behaviour under a Weyl transformation:

$\delta b(x, \rho)=b^{\prime}(x, \rho)-b(x, \rho)=-2 \sigma(x) \rho \partial_{\rho} b(x, \rho)+\stackrel{(0)}{\nabla}_{i}\left(b(x, \rho) a^{i}(x, \rho)\right)$.

Using the explicit expression (2.4), it is now straightforward to show that $b(x, \rho)$ satisfies the Wess-Zumino (WZ) consistency condition:

$$
\int \mathrm{d}^{d} x \sqrt{g_{(0)}}\left(\sigma_{1} \delta_{\sigma_{2}} b-\sigma_{2} \delta_{\sigma_{1}} b\right)=0 .
$$

We will see below that $b(x, \rho)$ may be considered as a generating function for Weyl anomalies in all (even) dimensions.

$\dagger$ We use the following curvature conventions: $\left[\nabla_{i}, \nabla_{j}\right] V_{k}=-R_{i j k}^{l} V_{l}$ and $R_{i j}=R_{i k j}{ }^{k}$. 
In order to explicitly use the Weyl transformation properties we expand the generating function $b(x, \rho)$ in a power series in $\rho$ :

$$
b(x, \rho)=\sum_{n=0}^{\infty} b_{n}(x) \rho^{n} .
$$

Using (3.6) the $\rho$ integrations can be performed explicitly and the boundary action $S$ expressed in terms of the $b_{n}$ :

$$
\kappa_{d+1}^{2} S=\sum_{n=0}^{\infty} \frac{l}{2 n-d} \int \mathrm{d}^{d} x \sqrt{g_{(0)}(x)} b_{n}(x) .
$$

For $b(x, \rho)$ evaluated on a solution $g(x, \rho)$ the coefficients $b_{n}(x)$ become scalar local functionals of $g_{(0)}$. The PBH transformation (3.4) gives the transformation of $b_{n}(x)$ under a Weyl transformation:

$$
\delta b_{n}=-2 n \sigma b_{n}+\nabla_{i}\left(\sum_{m=0}^{n-1} b_{m} a_{(n-m)}^{i}\right) .
$$

Explicitly, for the first few coefficients, we find

$$
\begin{aligned}
& \delta b_{0}=0, \\
& \delta b_{1}=-2 \sigma b_{1}+\frac{1}{2} l^{2} b_{0} \square \sigma, \\
& \delta b_{2}=-4 \sigma b_{2}-\frac{b_{0} l^{4}}{4(d-2)}\left[R^{i j} \nabla_{i} \nabla_{j} \sigma-\frac{1}{2} R \square \sigma\right] .
\end{aligned}
$$

$\delta b_{3}$, which is a rather unwieldy expression, contains the two arbitrary parameters $c_{1}$ and $c_{2}$ in $g_{(2) i j}$.

The Weyl variation determines to a certain extent the dependence of $b_{n}(x)$ on $g_{(0)}$. We will argue in the next section that local expressions for $b_{n}$ which satisfy (3.9) can always be found. We give here again only the first few explicit expressions:

$$
\begin{aligned}
b_{0} & =\text { constant } \\
b_{1} & =b_{0} \frac{l^{2}}{4(d-1)} R, \\
b_{2} & =\frac{l^{4} b_{0}}{32(d-2)(d-3)} E_{4}+c l^{4} C_{i j k l} C^{i j k l},
\end{aligned}
$$

where as before $C_{i j k l}$ is the Weyl tensor corresponding to $g_{(0)}$ in $d$ dimensions and $E_{2 n}$ is the Euler density, which is defined in $d=2 n$ dimensions as

$$
E_{2 n}=\frac{1}{2^{n}} R_{i_{1} j_{1} k_{1} l_{1}} \cdots R_{i_{n} j_{n} k_{n} l_{n}} l^{i_{1} j_{1} i_{2} j_{2} \ldots i_{n} j_{n}} \epsilon^{k_{1} l_{1} \ldots k_{n} l_{n}} .
$$

Once the product of the epsilon tensors has been expressed in terms of products of the metric, this expression makes sense in any dimension.

Again, starting from the second coefficient $b_{2}$ there are an increasing number of solutions to the homogeneous equations which enter with free parameters. These arbitrary coefficients and those appearing in the integration of $\delta g_{(n)}$ will appear in the expressions for $\delta b_{(n)}$ for $n>2 \uparrow$. In the next section we will discuss the various constraints on these coefficients following from their relation to trace anomalies.

$\dagger$ For instance, the homogeneous equation $\delta b_{3}=-6 \sigma b_{3}$ has three solutions. In addition, $b_{3}$ depends on the two parameters already present in the inhomogeneous piece of $\delta b_{3}$. 
The coefficients $b_{n}$ depend on the action through the constant $b_{0}$ and through the constants which enter through the solutions to the homogeneous equations. For $b_{0}$ we can obtain a general formula. Indeed, if we write the action (3.1) in the form

$2 \kappa_{d+1}^{2} S=\int \mathrm{d} \rho \mathrm{d}^{d} x \sqrt{G} f(R(G))=\frac{1}{2} l \int \mathrm{d} \rho \mathrm{d}^{d} x \rho^{-(d / 2)-1} \sqrt{g} f(R(G))$

then

$$
b_{0}=\left.l f(R)\right|_{\rho=0}=l f(\operatorname{AdS}),
$$

where in the latter expression $f$ is to be evaluated at the action of $A d S_{d+1}$ space with

$$
R_{\mu \nu \rho \sigma}=\frac{1}{l^{2}}\left(G_{\mu \rho} G_{\nu \sigma}-G_{\mu \sigma} G_{\nu \rho}\right) .
$$

To see this one simply has to realize that $\sqrt{g} f(R)$ has an expansion in positive powers of $\rho$ and that only the most singular (in $\rho$ ) contributions of $R_{\mu \nu \rho \sigma}$ contribute to $b_{0}$. It is straightforward to show that this contribution is as given in (3.14).

We see, therefore, that the effective action of the boundary metric has a structure determined to a large extent by the action of the Weyl transformations which in turn are fixed by the PBH transformation. The specific form of the bulk action manifests itself through a set of constants: $b_{0}$ which has the universal structure given by (3.13) and the coefficients of the terms involving the Weyl tensors in (3.8) and (3.10).

We remark that the general expressions (3.10), being based on symmetry considerations, do not assume that the action which gives the equation of motion which determines $g_{i j}(x, \rho)$ and the action which determines $b$ should be the same, provided both are diffeoinvariant. The explicit expression (3.13) for $b_{0}$ needs of course the identity of the two actions.

In the next section we will exploit the relation between the effective action and trace anomalies in order to constrain the ambiguities, on one hand, and to extract universal properties of the trace anomalies from the effective action, on the other hand.

\section{Trace anomalies and the effective action}

We start this section with a brief summary of the general structure of trace anomalies (cf [9-11]) and the appearance of trace anomalies in boundary actions [3].

The trace anomalies can be characterized by the anomalous Weyl variation of a diffeoinvariant effective action depending on a metric in $d=2 n$ dimensions. This action reflects the properties of a conformally invariant matter theory and it is obtained, in principle, by coupling the matter to a classical background metric and integrating the matter fields out.

In general, the effective action is a non-local functional of the metric. Its Weyl variation, however, representing an anomaly is necessarily local. The second variation of the action, i.e. the first variation of the anomaly, is a symmetric functional of the two infinitesimal Weyl parameters. This integrability condition is the Wess-Zumino condition the anomaly must satisfy.

The above stated structure offers a general classification of trace anomalies in any even dimension:

(a) There is always the type A anomaly whose expression is the Euler characteristic in the respective dimension. This type does not reflect any real logarithmic ultraviolet divergence. In dimensional regularization, however, out of the term in the effective action giving rise to it, a piece having a simple pole in $2 n-d$ multiplying the Euler characteristic, can be separated, giving a clear signature for this type of anomaly. 
(b) There is an increasing (with dimension) number of type B anomalies: they originate in true logarithmic divergences in correlators of lower order than the anomalous ones. Their signature is an expression which is exactly Weyl invariant. The simplest way to construct Weyl-invariant expressions is through the contraction of Weyl tensors but there are invariants starting with covariant derivatives of Weyl tensors, etc. In dimensional regularization, these terms appear directly in the effective action multiplying poles in $2 n-d$.

In addition there are cohomologically trivial local expressions, i.e. obtained by Weyl variations of local, Weyl non-invariant, pieces in the effective action. These terms, representing arbitrary real parts (subtraction constants) in the underlying matter theory, cannot contain any dynamical information and their explicit form depends on the regularization chosen.

Going now back to (3.7) we observe that $b_{n}$ appears multiplying a pole in $2 n-d$ : therefore $b_{n}$ is a trace anomaly in $d=2 n$ dimensions, i.e. it could be expanded as a linear combination of a type $\mathrm{A}$ and the type $\mathrm{B}$ appropriate to the dimension and cohomologically trivial terms. Indeed, as mentioned in the previous section, the $b_{n}$ 's satisfy the WZ condition, equation (3.5).

A first conclusion we could reach is that equation (3.8) always has a solution in terms of local expressions which are linear combinations of types A and B, and are cohomologically trivial. Of course this puts constraints on the terms which could appear on the right-hand side of (3.8).

We will be more interested, however, in the way we could extract universal information about trace anomalies from the effective action. The anomaly of type A is a clear candidate for this type of information. Indeed, as we remarked before, terms involving contractions of Weyl tensors (i.e. type B) appear with arbitrary coefficients in $b_{n}$; therefore we could not expect any simple, general expression for type B.

In order to isolate the general expression we are looking for in type A, we use the following simple observation: an exactly Weyl-invariant expression vanishes identically for a metric which is conformally flat, i.e. which has the form

$$
g_{(0) i j}=\exp (2 \phi) \delta_{i j}
$$

Therefore, the terms with arbitrary coefficients in the various recursion relations ("the homogeneous terms') will disappear. Consequently, the unambiguous solution of the equations for a metric of the form (4.1) will give us information about the surviving type A anomaly.

We will expand the solutions in powers of $\phi$ defined in (4.1). In particular, the Euler characteristic in $d=2 n$ starts with the $n$th power of $\phi$ :

$$
\begin{aligned}
E_{2 n} & \equiv \frac{1}{2^{n}} R_{i_{1} j_{1} k_{1} l_{1}} \ldots R_{i_{n} j_{n} k_{n} l_{n}} \epsilon^{i_{1} j_{1} \ldots i_{n} j_{n}} \epsilon^{k_{1} l_{1} \ldots k_{n} l_{n}} \\
& =n ! 2^{n}\left(\partial_{i_{1}} \partial_{j_{1}} \phi\right) \cdots\left(\partial_{i_{n}} \partial_{j_{n}} \phi\right) \delta_{j_{1} \ldots j_{n}}^{i_{1} \ldots i_{n}}+\mathcal{O}\left(\phi^{n+1}\right) .
\end{aligned}
$$

Now, our main problem is to isolate the contribution of the type A anomaly (proportional to the Euler characteristic) from the cohomologically trivial pieces. We rewrite the basic recursive relation (3.8) in an integrated form by multiplying it by an infinitesimal Weyl parameter $\sigma_{1}$, the variation being with a new Weyl parameter $\sigma_{2}$ :

$$
\begin{array}{r}
\int \mathrm{d}^{d} x \sqrt{g_{(0)}(x)} \sigma_{1} \delta_{2} b_{n}=-2 n \int \mathrm{d}^{d} x \sqrt{g_{(0)}(x)} \sigma_{1} \sigma_{2} b_{n} \\
-\int \mathrm{d}^{d} x \sqrt{g_{(0)}(x)} \nabla_{i} \sigma_{1} \sum_{m=0}^{n-1} b_{m} a_{(n-m)}^{i},
\end{array}
$$


where $a^{i}$ contains $\sigma_{2}$. For a metric of the form (4.1) and expanding in powers of $\phi$ it is easy to see that $b_{n}$ starts with a term containing $n$ powers of $\phi$ and that $a^{i}$ starts with terms as follows:

$$
\begin{aligned}
& a_{(1)}^{i}=\frac{1}{2} l^{2} \partial_{i} \sigma+\mathcal{O}(\phi), \\
& a_{(2)}^{i}=-\frac{1}{4} l^{4}\left(\partial_{i} \partial_{j} \phi\right) \partial_{j} \sigma+\mathcal{O}\left(\phi^{2}\right), \\
& a_{(3)}^{i}=\frac{1}{8} l^{6}\left(\partial_{i} \partial_{k} \phi\right)\left(\partial_{k} \partial_{j} \phi\right) \partial_{j} \sigma+\mathcal{O}\left(\phi^{3}\right) .
\end{aligned}
$$

Using induction it is straightforward to prove that (4.4) generalizes as expected, i.e. that the term of order $n$ has the form

$$
a_{(n)}^{i}=(-)^{n+1} \frac{l^{2 n}}{2^{n}}\left[\left(\partial^{2} \phi\right)^{n-1}\right]_{i j} \partial_{j} \sigma+\mathcal{O}\left(\phi^{n}\right) .
$$

Now, in an expansion in $\phi$ of $b_{n}$ the cohomologically trivial pieces come from the Weyl variation of a local expression containing $n+1$ powers of $\phi$. The variation replaces each field $\phi$ in turn by $\sigma_{1}$. Consequently, the result will be symmetric under the interchange of each $\phi$ with $\sigma_{1}$. The Euler characteristic multiplied by $\sigma_{1}$ does not share this symmetry property. Therefore, if we antisymmetrize equation (4.3), i.e. if from the equation multiplied by $n$ we subtract $n$ terms, where $\sigma_{1}$ was interchanged with each factor $\phi$ and with $\sigma_{2}$, respectively, we will be left on the left-hand side with the contribution of the Euler term only $\dagger$.

We can now specialize even further and pick one particular term in the Euler characteristic e.g. the contribution $n ! 2^{n}(\square \phi)^{n}$. This term will give a contribution $\sigma_{1} \square \sigma_{2}(\square \phi)^{n-1}$ to the integrand of the left-hand side of (4.3), antisymmetrized of course, and with a numerical coefficient following from our normalizations.

We can try to match this term with terms on the right-hand side of (4.3). From the explicit form of $a^{i}$ we see that all terms on the right-hand side except the last $(n-1)$ th one in the sum, will have two partial derivatives not contracted into a $\square$. In the last term, however, we have, after a partial integration, a contribution to the integrand of the form $\sigma_{1} \square \sigma_{2} b_{n-1}$ antisymmetrized. Again the antisymmetrization projects out from $b_{n-1}$ all contributions but the Euler one in $(n-1)$ th order in $\phi$. In particular, the $(\square \phi)^{n-1}$ contribution to $E_{2(n-1)}$ matches the left-hand side nicely.

Therefore, we obtained a recursion relation between the Euler contributions alone, in different orders. Reintroducing the numerical factors we left out in the previous argument, the recursion can be easily solved and we obtain for the Euler contribution the general formula

$$
b_{n}=\frac{l^{2 n} b_{0}}{2^{2 n}(n !)^{2}} E_{2 n}+\text { cohomologically trivial terms. }
$$

Using the expression (3.13) for $b_{0}$ evaluated for $d=2 n$ we now have a general formula for the type A trace anomaly in any even dimension corresponding to a given gravity action which admits an AdS solution. We have to think about the gravity action given abstractly as a polynomial in the curvature without specifying the dimension. The specific form of the action enters in determining the radius of the AdS solution and then in the evaluation of the action on the solution, to give $b_{0}$. All the factors depending on the dimension $d=2 n$ in the expression for the coefficient of the type A anomaly are then explicit.

As we remarked already in the previous section the symmetry considerations do not require that the action which determines $g$ and that which determines $b$ should be the same. The universal dependence on the dimension appears, however, only if the two actions are identified.

$\dagger$ The term with $\sigma_{1}$ interchanged with $\sigma_{2}$ automatically cancels the original term, this being the content of the WZ condition; we could have left it out and changed the normalization to $n-1$. 


\section{Discussion}

Through the PBH argument the Weyl transformations of the effective boundary theory can be understood to follow from the diffeomorphisms of the bulk theory. The dependence on the 'odd' variable $\rho$ in the bulk gets translated into the dependence on the dimension, $b(x, \rho)$ playing the role of a generating function for the type A trace anomalies in various dimensions.

The final result is very similar in spirit to the 'elliptic genus' which gives, through the 'descent equations', a unifying expression for the chiral anomaly in various dimensions. The elliptic genus gives an expression for the chiral anomaly of gauge theories with chiral fermions in various dimensions which have the same gauge group and representation content of the fermions. In this analogy there is, however, a part we really do not understand, i.e. which is the common feature of the conformal field theories in various dimensions whose trace anomalies are represented by the same gravitational action? It is tempting to conjecture that general properties such as, for example, the 'flow' as measured by the anomaly coefficient, are common in these theories. This would suggest that the type A anomaly coefficient in $d=4$ related to $c$ in $d=2$ gives the right generalization of the $c$-theorem as first proposed by Cardy [12].

Beginning with $b_{3}$ the Euler characteristic appears accompanied by cohomologically trivial terms which are determined by the recursion relations. We do not understand the physical significance, if any, of these terms. More generally, it would be interesting to study the exact mathematical structure of the 'cohomological' equations (2.4), (2.9) and (3.9).

Finally, the special role the type A anomaly plays in the discussion suggests that it should be singled out for the understanding of the non-leading $1 / N$ corrections in the AdS/CFT correspondence which are still not under control [13]. We postpone the discussion of this problem to a later publication.

\section{Acknowledgments}

This work was partially supported by GIF, the German-Israeli Foundation for Scientific Research, by the European Commission TMR programme ERBFMRX-CT96-0045, Minerva Foundation, the Center for Basic Interactions of the Israeli Academy of Sciences and the US-Israel Binational Science Foundation.

\section{References}

[1] Aharony O, Gubser S S, Maldacena J, Ooguri H and Oz Y 1999 Large N field theories, string theory and gravity Preprint hep-th/9905111

[2] Witten E 1998 Adv. Theor. Math. Phys. 2253

(Witten E 1998 Anti-de Sitter space and holography Preprint hep-th/9802150)

[3] Henningson M and Skenderis K 1998 J. High Energy Phys. JHEP07(1998)023 (Henningson M and Skenderis K 1998 The holographic Weyl anomaly Preprint hep-th/9806087)

[4] Aharony O, Pawelczyk J, Theisen S and Yankielowicz S 1999 Phys. Rev. D 60066001

(Aharony O, Pawelczyk J, Theisen S and Yankielowicz S 1999 A note on anomalies in the AdS/CFT correspondence Preprint hep-th/9901134)

[5] Penrose R and Rindler W 1986 Spinors and Spacetime vol 2 (Cambridge: Cambridge University Press) ch 9

[6] Brown J D and Henneaux M 1986 Central charges in the canonical realization of asymptotic symmetries: an example from three-dimensional gravity Commun. Math. Phys. 104207

[7] Graham C R 1999 Volume and area renormalizations for conformally compact Einstein metrics Preprint math.dg/9909042

Skenderis K and Solodukhin S N 1999 Quantum effective action from the AdS/CFT correspondence Preprint hep-th/9910023 
Bautier K 1999 Diffeomorphisms and Weyl transformations in AdS(3) gravity Preprint hep-th/9910134

Nishimura M and Tanii Y 1999 Local symmetries in the AdS(7)/CFT(6) correspondence Preprint hep-th/9910192

[8] Fefferman C and Graham R 1995 Conformal Invariants, Astèrisque p 95

[9] Bonora L, Pasti P and Bregola M 1986 Weyl cocycles Class. Quantum Grav. 3635

[10] Cappelli A, Friedan D and Latorre J I 1991 C theorem and spectral representation Nucl. Phys. B 352616

[11] Deser S and Schwimmer A 1993 Geometric classification of conformal anomalies in arbitrary dimensions Phys. Lett. B 309279

(Deser S and Schwimmer A 1993 Preprint hep-th/9302047)

[12] Cardy J L 1988 Is there a C theorem in four-dimensions? Phys. Lett. B 215749

[13] Nojiri S and Odintsov S D 1999 On the conformal anomaly from higher derivative gravity in AdS/CFT correspondence Preprint hep-th/9903033

Blau M, Narain K S and Gava E 1999 J. High Energy Phys. JHEP09(1999)018

(Blau M, Narain K S and Gava E 1999 On subleading contributions to the AdS/CFT trace anomaly Preprint hep-th/9904179) 